\title{
Accidental scrotal burns from paraquat while handling a patient
}

\author{
R Premaratna', B G N Rathnasena ${ }^{2}$ and H J de Silva ${ }^{1}$ \\ (Index words: paraquat, scrotal burns)
}

\begin{abstract}
The main risk of paraquat poisoning is from deliberate ingestion. Serious accidental or occupational poisoning is comparatively rare. We report two patients who had accidental exposure to paraquat, resulting in scrotal burns in both and systemic poisoning in one, while attending to a patient who had ingested paraquat for deliberate self harm.
\end{abstract}

\section{Introduction}

Paraquat is a contact herbicide used in over 130 countries, including Sri Lanka [1]. The main risk of paraquat poisoning is from deliberate ingestion, and the severity of complications is related to the dose and route of exposure [2]. Serious accidental or occupational poisoning is comparatively rare, and when it does occur, it is usually due to inappropriate handling of the product or inadequate initial treatment, especially following dermal exposure [2,3]. Paraquat poisoning remains a management dilemma; lack of an effective antidote is the main drawback, and its being a strong corrosive as well as being absorbed through intact skin adds to the challenge $[2,3]$.

There are a few reports of accidental exposure to paraquat due to leakage from spraying containers [4] or application of paraquat to the skin by mistake [5]. This causes severe burns or dermatitis of the affected skin, sometimes with systemic poisoning [3,6,7]. We report two patients who had accidental exposure to paraquat resulting in scrotal burns in both, and systemic poisoning in one. Poisoning was caused by exposure to the vomitus of a patient who had ingested paraquat for deliberate self-harm.

\section{Case report}

A 19-year old male was admitted to hospital after ingestion of about $250 \mathrm{ml}$ of paraquat for deliberate self-harm. He was accompanied to the hospital by his 17-year old younger brother and a friend who was 20 years old. The patient had severe burns in his mouth and tongue. He developed extensive subcutaneous emphysema around the neck and upper chest within 6 hours of admission, and died of respiratory failure within 8 hours.

Two days after the patient's death, both his brother and the friend presented to hospital with painful swelling of their scrotum. In the brother, there were severe burns of the scrotal skin sparing the penile skin (figure). The area was very tender and had severe excoriation and oozing. The friend had milder burns of the scrotum. They had brought the patient to hospital in a three-wheeler taxi. Both of them were wearing sarong. The patient had been lying across their laps while being brought to hospital, and had vomited on them several times. They had stayed with the patient in the clothes soaked with vomitus, for the next 8 hours until his death. They had not informed the medical staff about being exposed to the vomitus as they were unaware of the consequences of skin contamination with paraquat.

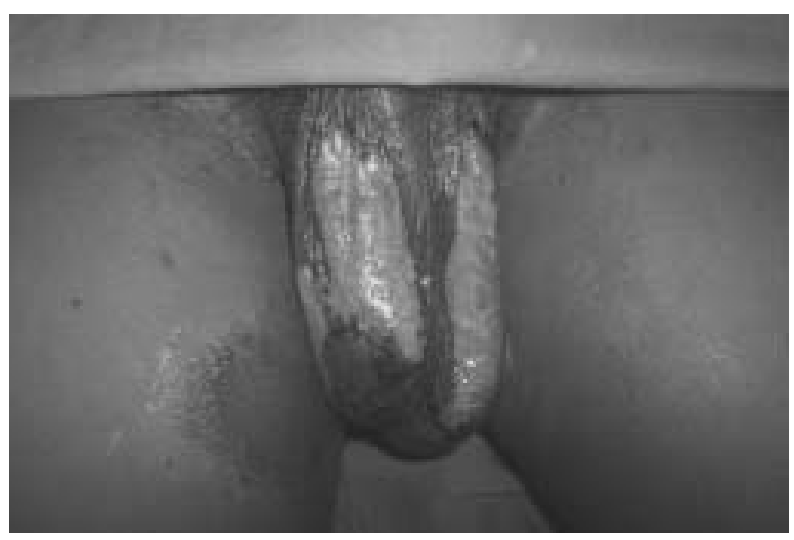

Figure. Scrotal burns due to paraquat

Both of them were kept under observation for possible renal, hepatic and respiratory complications. The brother developed mild derangements in his hepatic and renal function suggesting systemic absorption. His biochemical and clinical parameters returned to normal over the next two weeks on conservative management. His friend had no evidence of systemic poisoning with paraquat. In both, the scrotal skin healed without scarring over the next four

${ }^{1}$ Department of Medicine, Faculty of Medicine, University of Kelaniya, and ${ }^{2}$ Colombo North Teaching Hospital, Ragama. Correspondence: RP, e-mail <ranjan_premaratna@lycos.com>. Received 12 March 2008 and accepted 26 July 2008. Conflicts of interests: none declared. 
weeks with the local application of zinc sulphadiazine and oral amoxicillin.

\section{Discussion}

There are few reports of paraquat exposure where patients had accidental burns of the scrotum and perineal region due to mistaken application of paraquat in toilet containers [5], and due to leaks in spraying equipment used in agriculture [4]. In these cases, in addition to scrotal burns, significant systemic toxicity also occurred, possibly due to high vascularity of the scrotal skin. In this report, the two patients described were accidentally exposed to paraquat in the vomitus of a patient who had ingested it, while attending to him. The vomitus had seeped through their clothing causing scrotal burns. The fact that they did not immediately remove the contaminated clothes would have aggravated the burns.

We emphasise the importance of precautions that should be taken by persons attending to those who have ingested poison. This is especially applicable to patients who have ingested corrosive substances and agrochemicals which could cause severe burns and systemic toxicity by absorption through the skin.

\section{References}

1. Fernando R, de Silva DGH, Amarasena TSD. An unusual case of fatal accidental paraquat poisoning. Forensic Science International 1990; 44: 23-6.

2. Bismuth C, Hall AH; eds. Paraquat poisoning: mechanisms, prevention and treatment. New York: Marcel Dekker, 1995.

3. Smith JG. Paraquat poisoning by skin absorption: a review. Human Toxicology 1988; 7:15-9.

4. Ronnen M, Klin B, Suster S. Mixed diquat/paraquat-induced burns. International Journal of Dermatology 1995; 34: 23-5.

5. Withers EH, Madden JJ Jr, Lynch JB. Paraquat burn of the scrotum and perineum. Journal of the Tennessee Medical Association 1979; 72:109.

6. Botella R, Sastre A, Castells A. Contact dermatitis to paraquat. Contact Dermatitis 1985; 13: 123-4.

7. Wohlfhart DJ. Fatal paraquat poisoning after skin absorption. Medical Journal of Australia 1982; 1: 512-3. 